\title{
Avaliação e mensuração da sutura palatina mediana por meio da radiografia oclusal total digitalizada em pacientes submetidos à expansão rápida maxilar
}

Sandra Maria Nobre David*, Julio Cezar de Melo Castilho**, Cristina Lucia Feijó Ortolani***,

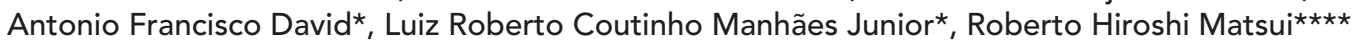

\section{Resumo}

Objetivos: avaliar e mensurar a sutura palatina mediana por meio de radiografias oclusais totais de maxila digitalizadas, antes e depois da sua disjunção. Métodos: a amostra constou de 17 pacientes, com idades entre 7 e 22 anos. Radiografias oclusais totais da maxila foram executadas antes e depois da abertura da sutura palatina mediana, e digitalizadas em scanner HP Scanjet 6110 C com adaptador de transparências HPC 62616100 C, utilizando-se o programa Deskscan II. Para a avaliação e medição, foi utilizado o programa Radioimp ${ }^{\circledR}$ (Radiomemory, MG/Brasil). Na análise estatística, foram utilizados a média, o desvio-padrão, o coeficiente de variação e os testes " $t$ " e ANOVA. Conclusões: após os resultados, foi possível concluir que (1) na região dos incisivos, houve uma abertura palatina mediana estatisticamente significativa; (2) houve abertura de diastema entre os incisivos centrais superiores em torno de 69,37\% dos casos; (3) houve uma maior abertura da sutura palatina mediana na região a $10 \mathrm{~mm}$ a partir da crista para posterior, em comparação com a região a $3 \mathrm{~mm}$ para posterior do parafuso expansor; (4) na região a $3 \mathrm{~mm}$ para posterior do parafuso expansor houve uma abertura de $35,97 \%$, e na região a $10 \mathrm{~mm}$ para posterior da crista uma abertura de $69,37 \%$.

Palavras-chave: Disjunção. Sutura palatina mediana. Radiografia digitalizada.

\section{INTRODUÇÃO E REVISÃO DA LITERATURA}

A sutura palatina mediana pode ser aberta a uma extensão suficiente para causar um alargamento significativo da maxila no sentido transversal, fazendo parte de um procedimento ortopédico/ortodôntico.
Com o propósito de normalizar essas situações, a expansão rápida da maxila compreende um recurso clínico incorporado integralmente à mecanoterapia contemporânea, independentemente da técnica empregada. A prova disso está no grande número de aparelhos expansores, divulgados na

* Doutores em Biopatologia Bucal, área Radiologia Odontológica, Unesp / São José dos Campos.

** Especialista, mestre e doutor em Radiologia Odontológica pela USP. Professor livre-docente da disciplina de Radiologia Odontológica do curso de

*** Especialista, mestre e doutora em Ortodontia pela USP. Professora titular da disciplina de Ortodontia da Faculdade de Odontologia da UNIP - Unidades Campinas e São Paulo. Coordenadora do curso de especialização em Ortodontia de UNIP - Unidade Campinas. Professora doutora do mestrado da UNIP - Unidade São Paulo.

**** Mestre em Ortodontia da Faculdade de Odontologia da UNIP - Unidade São Paulo. 
literatura, para a abertura da sutura palatina mediana ou disjunção palatina.

Existem inúmeros trabalhos publicados a respeito da abertura da sutura palatina mediana, bem como de todas as suturas circum-maxilares, após o procedimento mecânico funcional de aparelhos que propiciam esse resultado.

$\mathrm{Na}$ primeira referência ao procedimento de abertura da sutura palatina mediana que $\mathrm{Haas}^{9}$ executou, em um indivíduo do gênero feminino de 14 anos de idade, ele adaptou um dispositivo com um parafuso transversalmente à abóbada palatina, cujas extremidades forçavam o primeiro e o segundo pré-molares de um lado e apenas o segundo pré-molar do outro lado. A paciente foi orientada a girar o parafuso duas vezes ao dia e, ao final de duas semanas, a maxila foi alargada, deixando um diastema entre os incisivos centrais superiores.

Haas $^{10}$, em uma amostragem de três pacientes e observações clínicas em mais de 80 casos submetidos à expansão da sutura palatina mediana, notou que essa se separa, surgindo um diastema entre os incisivos centrais superiores.

Esse mesmo autor ${ }^{8}$ postulou que a sutura palatina mediana se abre e é paralela anteroposteriormente e triangular inferoposteriormente, com o vértice voltado para a cavidade nasal. As raízes dos dentes divergem mais do que as coroas dentárias, durante a ativação do parafuso. Cessada a ativação, as raízes continuam a divergir, enquanto as coroas inclinam-se rapidamente em direção à linha média e, quando as coroas se contatam, a tensão contínua das fibras causa a convergência das raízes dentárias para além de sua inclinação axial original. O processo alveolar se move lateralmente, enquanto o processo palatino se abaixa em sua margem livre.

Silva Filho, Montes e Torely ${ }^{15}$ concluíram que a mordida cruzada posterior deve ser tratada desde cedo e que expansão rápida maxilar pode ser feita em pacientes com dentadura decídua, mista ou permanente. Avaliaram a eficiência da expansão rápida da maxila em 32 crianças pela inspeção de radiografias posteroanteriores (PA). Os resultados confirmaram que a abertura triangular da área frontoalveolar é maior do que em outras partes da sutura palatina.

Iseri et al. ${ }^{11}$ avaliaram o efeito biomecânico da expansão rápida da maxila sobre o complexo craniofacial, utilizando um modelo tridimensional de elementos finitos (MEF) do esqueleto craniofacial. A definição do MEF tridimensional foi baseada em uma leitura de tomografia computadorizada (TC) do crânio de um indivíduo do gênero masculino com 12 anos de idade. As fotos da TC foram digitalizadas e convertidas no modelo de elementos finitos por meio de um procedimento desenvolvido para esse estudo. A estrutura final compreendeu 2.270 elementos densos, em forma de concha, com 2.120 nódulos. A resposta mecânica, considerando-se o deslocamento e a resistência de Von Mises, foi avaliada expandindo-se a maxila mais de $5 \mathrm{~mm}$ em ambos os lados. Pela vista oclusal, as duas metades da maxila estavam separadas, quase de forma paralela, durante as expansões de 1,3 e $5 \mathrm{~mm}$. Observou-se uma expansão maior na área dentoalveolar e um decréscimo gradual nas estruturas superiores A largura do assoalho da cavidade nasal aumentou notadamente. Entretanto, a parte posterossuperior da cavidade nasal movimentouse levemente para medial. Não foi observado deslocamento nos ossos parietal, frontal e occipital. Foram observados altos níveis de resistência nas regiões dos caninos e dos molares superiores, na parede lateral da cavidade nasal inferior, e nos ossos nasais e zigomáticos, com uma concentração de resistência mais elevada nas lâminas pterigoides do osso esfenoide na região próxima à base craniana.

Cross e McDonald ${ }^{5}$ compararam as dimensões transversais das estruturas esqueléticas, dentárias e nasais de um grupo de pacientes com estreitamento maxilar, antes e após a expansão rápida da maxila (ERM), com um grupo controle nãotratado, utilizando radiografias cefalométricas posteroanteriores. $\mathrm{O}$ material constituiu-se de 25 
cefalogramas de crianças com mordida cruzada posterior e 25 controles, pareados quanto ao gênero e idade (13 anos e 11 meses). A ERM produziu alterações pequenas, mas estatisticamente significativas na largura maxilar, largura do molar superior e inferior, largura entre os ápices dos incisivos centrais superiores e largura intranasal. Há alguma evidência de que o padrão de expansão produzido pela ERM irá variar dependendo da idade e da maturidade do indivíduo.

Franchi et al. ${ }^{6}$ investigaram os efeitos, a curto e longo prazo, induzidos pela expansão rápida da maxila na forma das estruturas maxilares e circum-maxilares por meio da análise de lâmina fina. A amostra consistiu de 42 pacientes que foram comparados com uma amostra controle de 20 indivíduos. Os indivíduos sofreram ERM tipo Haas $^{9}$, seguida de terapia com aparelho fixo. Foram analisados os cefalogramas posteroanteriores, de cada indivíduo tratado, no Tl (pré-tratamento), T2 (pós-expansão imediata) e T3 (observação a longo prazo). $\mathrm{O}$ estudo avaliou as alterações de forma nas regiões maxilar, nasal, zigomática e orbital. Os autores observaram mudanças e concluíram que a ERM com o aparelho de Haas ${ }^{9}$ parece ser um meio terapêutico eficiente para induzir alterações favoráveis permanentes na forma do complexo nasomaxilar.

Jafari, Shetty e Kunar ${ }^{12}$, com o objetivo de analisar os padrões de distribuição de tensão dentro do complexo craniofacial durante a ERM, geraram um modelo de elementos finitos de um crânio humano jovem, usando dados copiados de tomografias computadorizadas de um crânio seco. O modelo foi, então, submetido a uma expansão da maxila, simulando a situação clínica. O padrão tridimensional de deslocamento e distribuição de tensão foi então analisado. O deslocamento lateral máximo foi de $5,313 \mathrm{~mm}$ na região dos incisivos centrais superiores. As porções inferiores das lâminas pterigoides também foram deslocadas lateralmente. As lâminas pterigoides próximas à base do crânio sofreram um deslocamento mínimo. O deslocamento máximo para a frente foi de $1,077 \mathrm{~mm}$, observado na região da borda anteroinferior do septo nasal.

\section{PROPOSIÇÃO}

Existem, na literatura, vários trabalhos científicos que analisaram, por meio de radiografias oclusais totais da maxila, a forma como se apresenta a sutura palatina mediana antes e depois da sua abertura.

A proposta do presente estudo é visualizar a sutura palatina mediana por meio de radiografias oclusais digitalizadas em momentos diferentes (antes e imediatamente após a abertura da sutura). Assim, é possível evidenciar detalhadamente sua forma, gerando uma imagem mais definida, a qual foi avaliada e mensurada pelo computador. Desse modo, torna-se possível acrescentar essa informação contemporânea à clínica radiográfica, ortodôntica e ortopédica funcional.

\section{MATERIAL E MÉTODOS}

\section{Material}

Amostra

A amostra foi constituída por 17 pacientes com idades entre 7 e 22 anos - dos arquivos da Disciplina de Radiologia Odontológica da Faculdade de Odontologia da Unesp / São José dos Campos - que necessitaram submeter-se à disjunção palatina por meio da expansão rápida da maxila com a utilização do disjuntor tipo Haas ${ }^{9}$.

\section{Traçados}

Foi utilizado o seguinte material para a obtenção dos traçados da oclusão:

a) radiografia oclusal;

b) computador Pentium IV;

c) adaptador gráfico super VGA padrão VESA;

d) monitor de vídeo super VGA colorido;

e) unidade de CD-ROM;

f) scanner HP Scanjet modelo 6100C;

g) leitor de transparências HPC 6261 6100C;

h) impressora tipo HP PSC1210; 
i) mouse serial Genius;

j) programa Radiomp/Radiocef Studio ${ }^{\circledR}(\mathrm{Ra}-$ diomemory, Belo Horizonte/MG).

\section{Métodos}

\section{Obtenção das radiografias oclusais}

As radiografias oclusais que foram utilizadas fazem parte dos arquivos da disciplina de Radiologia Odontológica da FOSJC - Unesp.

A instalação do aparelho disjuntor do tipo Haas foi feita logo após o início do tratamento. As ativações do aparelho duraram, em média, de 10 a 15 dias, com duas ativações diárias - sendo 2/4 de volta pela manhã e 2/4 de volta à noite nas crianças, e 1/4 de volta pela manhã e 1/4 de volta à noite nos adolescentes tardios e adultos - até conseguir-se a disjunção desejada. Após essa fase, o parafuso expansor foi estabilizado, com a fixação do mesmo por um período de 120 dias.

As radiografias oclusais totais da maxila foram executadas em duas fases da pesquisa: a primeira obtida na fase de pré-disjunção, logo após a instalação do aparelho, e a segunda imediatamente após a constatação clínica da disjunção.

As radiografias oclusais foram examinadas em negatoscópio com luz adequada e com máscara escura, para melhor visualização da imagem, sendo analisadas uma a uma, respeitando-se os critérios de análise radiográfica de cada fase:

1) Presença visível da sutura, mesmo que sinuosa, e ausência de áreas patológicas e/ou alterações radiolúcidas ou radiopacas no osso maxilar que comprometessem o tratamento.

2) Presença da abertura da sutura palatina mediana, expressa por área radiolúcida separando os segmentos ósseos maxilares.

Digitalização das tomografias lineares para a realização do traçado computadorizado

As radiografias oclusais foram digitalizadas no scanner, utilizando-se seu respectivo software (DeskScan II) para captura de imagens. Todas as imagens foram capturadas mantendo a resolução fixa de 75 dpi e escala de 100\% no modo sharp black and white photo. As imagens foram arquivadas no formato PCX.

\section{Traçado computadorizado}

Para a análise das radiografias oclusais, foram utilizados os seguintes pontos anatômicos disponibilizados pelo software Radioimp ${ }^{\circledR}$ para determinar segmentos (Fig. 1) e realizar as análises inicial e final das radiografias oclusais:

A) distância entre os incisivos centrais superiores na borda incisal;

B) distância entre as cristas alveolares na sutura palatina mediana;

C) abertura na distância de $10 \mathrm{~mm}$ da crista para posterior na sutura palatina mediana;

D) abertura na distância de $3 \mathrm{~mm}$ do segmento distal do parafuso expansor para posterior na sutura palatina mediana.

Esse programa oferece recursos para facilitar a visualização das estruturas no momento da marcação dos pontos para o traçado, tais como: alterações de brilho e contraste, ampliação ou redução da imagem, realce de bordas, pseudocoloração e inversão da imagem (Fig. 2). O programa disponibiliza ferramentas pré-determinadas e possibilita a criação de traçados, chamados de traçados individualizados, que foram utilizados para a confecção de um novo traçado (Fig. 3).

\section{Método estatístico}

Para avaliar os dados obtidos na medição das tomografias lineares, foi efetuado um estudo de confiabilidade dos métodos de mensuração utilizando-se:

a) média, desvio-padrão e coeficiente de variação;

b) apresentação do teste " $t$ " pareado;

c) ANOVA.

\section{RESULTADOS}

Verificou-se, aplicando o teste ANOVA para os valores das distâncias entre os incisivos, valores 


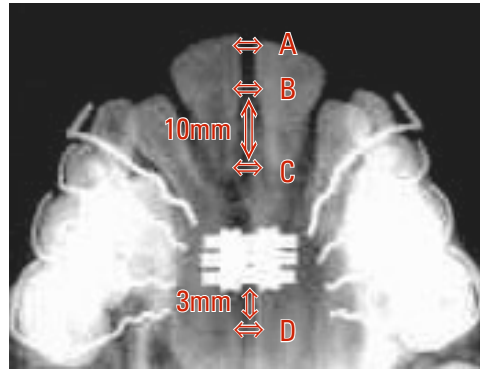

FIGURA 1 - Segmentos anatômicos utilizados na análise das radiografias.

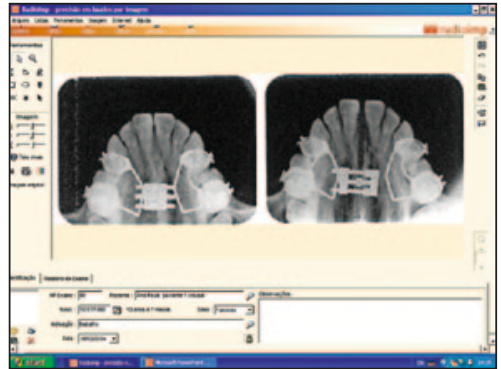

FIGURA 2 - Software Radiomp/Radiocef Stu$\mathrm{dio}^{\oplus}$.

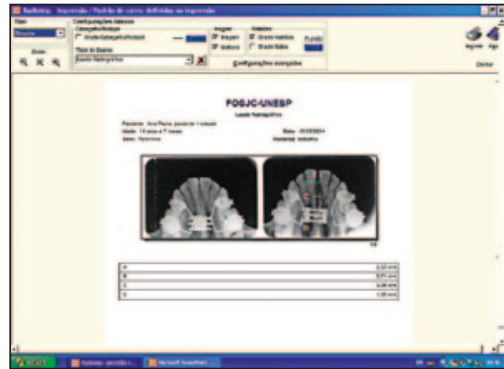

FIGURA 3 - Software Radioimp com as mensurações das radiografias oclusais. iniciais de $0,47 \pm 0,55 \mathrm{~mm}$ e pós-tratamento de $1,36 \pm 0,81 \mathrm{~mm}$, detectando-se uma diferença estatisticamente significativa ( $\mathrm{p}$-valor $=0,001)$ para o intervalo de confiança de 95\% (Gráf. 1).

Já para os valores correspondentes de abertura em crista, em $10 \mathrm{~mm}$ a partir da crista, e em $3 \mathrm{~mm}$ a partir do parafuso, utilizando o teste ANOVA

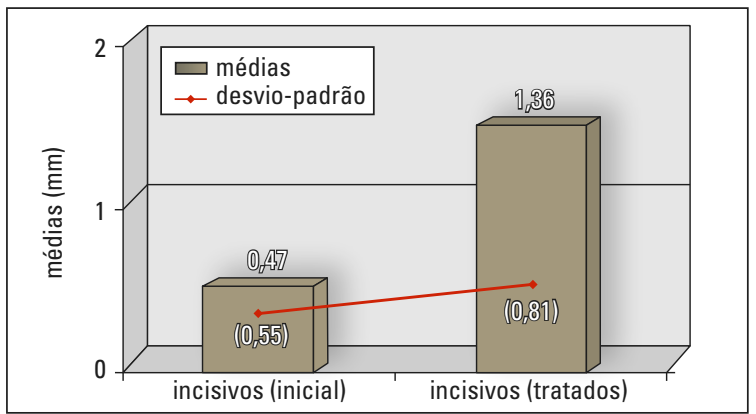

GRÁFICO 1 - Gráfico dos valores médios da abertura entre os incisivos antes e após a disjunção.

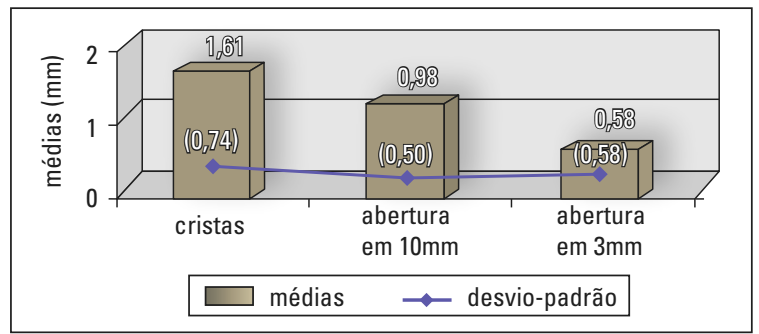

GRÁFICO 2 - Gráfico dos valores médios da abertura em crista, em 10mm e em $3 \mathrm{~mm}$. com $\alpha=5 \%$, notou-se valores médios de $1,61 \pm$ $0,74 \mathrm{~mm}, 0,98 \pm 0,50 \mathrm{~mm}$ e $0,58 \pm 0,58 \mathrm{~mm}$, respectivamente, sendo observada uma diferença estatisticamente significativa ( $\mathrm{p}$-valor $=0,00$ ) para $\mathrm{p} \geq 0,05$ (Gráf. 2). Para a análise do valor relativo (\%) de abertura do diastema, verificou-se que, em média, ocorreu uma abertura favorável de 69,38\%.

Para os valores relativos (\%) das aberturas nos segmentos em 10 e em $3 \mathrm{~mm}$, pode-se verificar que os valores médios apresentaram $69,37 \%$ e $35,97 \%$, respectivamente, de abertura em relação à abertura da crista. A diferença entre as porcentagens de $10 \mathrm{~mm}$ e $3 \mathrm{~mm}$ foi de 33,40\% (Gráf. 3).

Analisando os resultados, pode-se verificar que a porcentagem de abertura para o segmento de $10 \mathrm{~mm}$ para posterior foi próxima à dos incisivos, considerando que uma leve disjunção já é um fator significativo, se comparado aos valores quase nulos do estágio inicial (Gráf. 3).

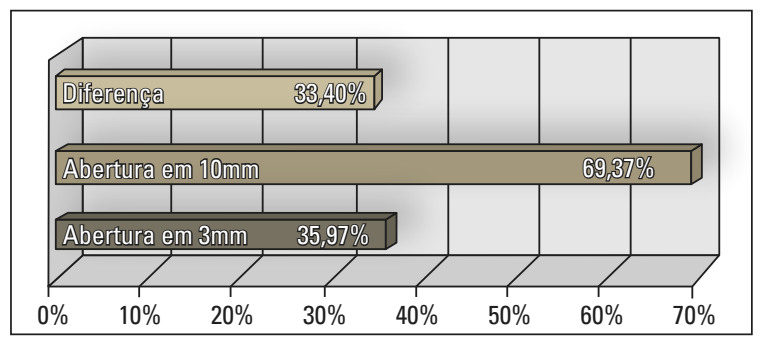

GRÁFICO 3 - Gráfico das porcentagens da quantidade de abertura da sutura palatina mediana nas diferentes regiões. 


\section{DISCUSSÃO}

A sutura palatina mediana pode ser aberta, por meio de disjuntores, no sentido transversal, fazendo parte de um procedimento ortopédico/ortodôntico. Essa abertura já foi descrita por diversos autores $^{4,5,7-10,12,13,15,16,17}$ e os resultados do presente estudo também a confirmam, nas regiões avaliadas.

A abertura do diastema entre os incisivos centrais superiores foi bem evidenciada em nossos resultados, com uma média inicial de $0,47 \pm$ $0,55 \mathrm{~mm}$ e após a disjunção de $1,36 \pm 0,81 \mathrm{~mm}$, verificando-se uma diferença estatisticamente significativa entre elas ( $\mathrm{p}$-valor $=0,001$ ) para o intervalo de confiança de $95 \%$, concordando com os trabalhos de $\operatorname{Haas}^{8,9,10}$, Jafari, Shetty e Kunar ${ }^{12}$.

Essa abertura do diastema entre os incisivos centrais superiores foi, para a análise do valor relativo (\%) de abertura do diastema, uma abertura favorável de $69,38 \%$, não alcançando a totalidade devido à presença de alguns pacientes adultos fazendo parte da amostra, nos quais houve certa dificuldade na obtenção da abertura da sutura palatina mediana. Isso vem de encontro à opinião de Biederman $^{3}$, que relatou que o fator idade deve ser considerado no tratamento com expansão rápida maxilar. Depois da fusão dos ossos esqueléticos (sinostoses), o prognóstico torna-se precário e é provável que todas as suturas faciais sejam envolvidas, em maior ou menor grau, sendo que as mais importantes são a palatina mediana, a frontonasal, a zigomatomaxilar e a zigomatotemporal, nessa ordem, enquanto as respostas teciduais variam com a idade do paciente $\mathrm{e}^{1,2}$.

Quando foi avaliada a abertura da sutura palatina mediana nas regiões da crista, a $10 \mathrm{~mm}$ da crista para posterior na sutura palatina mediana, e a $3 \mathrm{~mm}$ do segmento distal do parafuso expansor para posterior na sutura palatina mediana, observou-se os valores de $1,61 \pm 0,74 \mathrm{~mm}, 0,98 \pm 0,50 \mathrm{~mm}$ e 0,58 $\pm 0,58 \mathrm{~mm}$, respectivamente, sendo observada uma diferença estatisticamente significativa ( $\mathrm{p}$-valor $=0,00)$ para $p \geq 0,05$, ou seja, houve uma maior abertura na região da crista em comparação com os segmentos mais posteriores, mostrando uma conformação triangular de abertura da sutura palatina mediana, com a base do triângulo voltada para a região anterior e o vértice para posterior. Esses resultados estão de acordo com os encontrados por Krebs $^{13}$, Haas ${ }^{8}$ e Silva Filho, Montes e Torely ${ }^{15}$.

Do mesmo modo, complementando essa avaliação, ao calcular a porcentagem de abertura nas diferentes regiões, verificou-se que, para os valores relativos (\%) das aberturas em 3 e $10 \mathrm{~mm}$, pode-se detectar que os valores médios apresentaram 35,97 e 69,37\%, respectivamente, de abertura em relação à abertura da crista. A diferença entre as porcentagens das regiões em 10 e $3 \mathrm{~mm}$ foi de 33,40\%.

O enorme avanço tecnológico oriundo do desenvolvimento de hardwares e softwares aplicados à Odontologia, e das novas formas de aquisição de imagens pela introdução de detectores eletrônicos, fez da Radiologia contemporânea a área de maior e mais rápido desenvolvimento e alcance, desde a descoberta dos raios X. Esses avanços na aquisição de imagens evidenciam o uso do computador tanto no seu processamento como em sua análise, tornando-o superior aos métodos subjetivos usados anteriormente ${ }^{14}$, o que concorda com os resultados obtidos nesse trabalho, pois foram usados amplamente todos os recursos de digitalização e ferramentas disponíveis no programa adotado para as mensurações.

\section{CONCLUSÕES}

Com base nos resultados obtidos, chegou-se às seguintes conclusões:

1) $\mathrm{Na}$ região dos incisivos, houve uma abertura palatina mediana estatisticamente significativa.

2) Houve abertura de diastema entre os incisivos centrais superiores em torno de $69,38 \%$ dos casos.

3) Houve uma maior abertura da sutura palatina mediana na região a $10 \mathrm{~mm}$ a partir da crista para posterior, em comparação com a região a $3 \mathrm{~mm}$ para posterior do parafuso expansor.

4) Houve uma abertura de $35,97 \%$ na região 
a $3 \mathrm{~mm}$ para posterior do parafuso expansor, e de $69,37 \%$ de abertura na região a $10 \mathrm{~mm}$ para posterior da crista.

\title{
Evaluation and measurement of midpalatal suture through the digitalized occlusal radiography in patients submitted to rapid maxillary expansion
}

\begin{abstract}
Aim: to evaluate and to measure the midpalatal suture through digitalized occlusal radiographies before and after its disjunction. Methods: The sample was comprised by 17 patients, with ages from 7 to 22 years. Occlusal radiographies of these patients were obtained before and after the suture's opening. The occlusal radiographies were digitalized in a HP Scanjet 6100 C scanner with the HPC 62616100 C transparencies adaptor and the Deskscan II software. The statistical analysis used average, standard deviation, variation coefficient, " $\mathrm{t}$ " test and ANOVA. Conclusions: After the results, it was possible to conclude that: (1) statistically, there was a significant midpalatal opening in the incisors area; (2) there was an opening in the diastema between upper central incisors in about $69.37 \%$ of the cases; (3) there was a bigger midpalatal suture opening in the $10 \mathrm{~mm}$ area from the crest to posterior than in the $3 \mathrm{~mm}$ area to posterior from the screw; $(4)$ in the $3 \mathrm{~mm}$ area to posterior from the screw there was an opening of $35.97 \%$ and in the $10 \mathrm{~mm}$ area to posterior of the crest there was an opening of $69.37 \%$.
\end{abstract}

Keywords: Disjunction. Midpalatal suture. Digitalized radiography.

\section{REFERÊNCIAS}

1. BACCETTI, T. et al. Treatment timing for rapid maxillary expansion. Angle Orthod., Appleton, v. 1, no. 5, p. 343-350, Oct. 2001.

2. BELL, R. A. A review of maxillary expansion in relation to rate of expansion and patient's age. Am. J. Orthod., St. Louis, v. 81 , no. 1, p. 32-37, June 1982

3. BIEDERMAN, W. Rapid correction of Class III malocclusion by midpalatal expansion. Am. J. Orthod., St. Louis, v. 63, no. 1, p. 47-55, 1973.

4. BISHARA, S. E.; STALEY, R. N. Maxillary expansion: Clinical implications. Am. J. Orthod. Dentofacial Orthop., St. Louis, v. 91, no. 1, p. 3-14, Jan. 1987.

5. CROSS, D. L.; MCDONALD, J. P. Effects of rapid maxillary expansion on skeletal, dental and nasal structures: A posteroanterior cephalometric study. Eur. J. Orthod., Oxford, v. 22, no. 5, p. $519-528,2000$.

6. FRANCHI, L.; BACETTI, T.; CAMERON, C. G.; KUTCIPAL, E. A McNAMARA JR., A. Thin-plate spline analysis of the short and long-term effects of rapid maxillary expansion. Eur. J. Orthod. Oxford, v. 24, no. 2, p. 143-150, 2002

7. HAAS, A. J. Long-term posttreatment evaluation of rapid palatal expansion. Angle Orthod., Appleton, v. 50, no. 3, p. 189-217, 1980

8. HAAS, A. J. Palatal expansion: Just the beginning of dentofacial orthopedics. Am. J. Orthod., St. Louis, v. 57, no. 3, p. 219-255, Mar. 1970

9. HAAS, A. J. Rapid expansion of the maxillary dental arch and nasal cavity by opening the midpalatal suture. Angle Orthod. Appleton, v. 31, no. 2, p. 73-90, Apr. 1961.

10. HAAS, A. J. The treatment of maxillary deficiency by opening the midpalatal suture. Angle Orthod., Appleton, v. 35, no. 3, p. 200-217, July 1965.

11. ISERI, H.; TEKKAYA, A. E.; OZTAN, O.; BILGIÇ, S. Biomechanics effects of rapid maxillary expansion on the craniofacial skeleton, studied by the finite element method. Eur. J. Orthod., Oxford, v. 20, no. 4, p. 347-356, 1998.
12. JAFARI, A.; SHETTY, K. S.; KUNAR, M. Study of stress distribution and displacement of various craniofacial structures following application of transverse orthopedic forces - a three dimension FEM study. Angle Orthod., Appleton, v. 73, no. 1, p. 12-20, 2003.

13. KREBS, A. Midpalatal suture expansion studied by the implant method over a seven-year period. Rep. Congr. Eur. Orthod. Soc., London, v. 40, p. 131-142, 1964.

14. MOL, A. Image processing tools for dental applications. Dent. Clin. North Am., Philadelphia, v. 44, no. 2, p. 299-318, Apr. 2000.

15. SILVA FILHO, O. G.; MONTES, L. A.; TORELY, F. L. Rapid maxillary expansion in the deciduous and mixed dentition evaluated through posteroanterior cephalometric analysis. Am. J. Orthod. Dentofacial Orthop., St. Louis, v. 107, no. 3, p. 268-275, Mar. 1995.

16. SPOLYAR, J. L. The design, fabrication and use of a fullcoverage bonded rapid maxillary expansion appliance. Am. J. Orthod., St. Louis, v. 86, no. 2, p. 136-145, Aug. 1984.

17. TIMMS, D. J. A study of basal movement with rapid maxillary expansion. Am. J. Orthod., St. Louis, v. 77, p. 500-507, May 1980. 